\title{
Integración de la gestión de la calidad con las buenas prácticas de manufactura en tres empresas farmacéuticas de inyectables en Bogotá, D. C.
}

Integration of quality management with good manufacturing practices in three pharmaceutical firms producing products for injection in Bogota, D.C.

Integração da gestão da qualidade com as boas práticas de fabricação em três empresas farmacêuticas de soluções injetáveis em Bogotá, D.C.

Recibido: 16 de noviembre de 2018 Revisado: 11 de febrero de 2019 Aceptado: 8 de marzo de 2019

Lu* Adriana Cárdenas Aristizábal***

Universidad Santo Tomás, Colombia

Cómo citar: Cárdenas Aristizábal, L. A. (2019). Integración de la gestión de la calidad con las buenas prácticas de manufactura en tres empresas farmacéuticas de inyectables Bogotá, D. C. Signos. Investigación en Sistemas de Gestión, 11(2), 131-153. Doi: https://doi.org/10.15332/24631140.5086

\footnotetext{
* Artículo de investigación.

** Magíster en Calidad y Gestión Integral, especialista en Administración de Empresas, química farmacéutica. Bogotá, Colombia. Correo electrónico: adry.cardenas@gmail.com. Orcid: https://orcid.org/0000-0002-5980-0964
} 


\section{RESUMEN}

Los laboratorios farmacéuticos en Colombia deben cumplir con reglamentaciones sanitarias tomadas de estándares internacionales, como las BPM, relacionadas con la producción y el control de calidad de los medicamentos. Sin embargo, en esas organizaciones se evidencia poca integración de los procesos. En este sentido, el objetivo de la investigación fue indagar la percepción de los empleados sobre el manejo actual de los sistemas de gestión en dichas organizaciones, las oportunidades de mejora y los aspectos de la gestión del riesgo y del conocimiento que aportan en la integración de esas normas para construir una propuesta de integración. Para ello se realizó un estudio mixto con alcance descriptivo. Se realizaron entrevistas semiestructuradas al personal directivo y dos encuestas, una al personal de nivel táctico y otra al de nivel operativo. Se logró generar una propuesta de integración del sistema de gestión de la calidad con las BPM, la gestión del riesgo y la del conocimiento, basada en el PHVA. Se concluye que la gestión del conocimiento brinda un aporte en el adecuado funcionamiento de la gestión de la calidad y las BPM y que también la gestión del riesgo es un facilitador para el cumplimiento de los objetivos organizacionales y de calidad, cuando es enfocada en los aspectos más críticos de la organización y de los procesos de fabricación. De esta manera se aumenta la seguridad a los pacientes y disminuyen las reacciones adversas, quejas, desviaciones o los retiros de producto del mercado.

Palabras clave: sistema de gestión de la calidad, ISO 9001:2015, BPM, gestión del riesgo, gestión del conocimiento, sistemas integrados.

\section{ABSTRACT}

Pharmaceutical laboratories in Colombian must comply with five health-related rules taken from international standards, such as those GMPs related to the production and quality control of medicinal drugs; however, a lack of process integration is observed in these organizations. In this regard, the objective of this research consisted of determining the employees' perception of the current implementation of management systems in the different organizations, improvement opportunities, and the aspects related to risk and knowledge management, which contribute with the integration of these standards, to develop an integration proposal. For this purpose, a mixed descriptive study was conducted. Semi-structured interviews with the directive staff and two surveys, one to the tactic personnel and another with operational personnel, were carried out. A proposal was developed for the integration of the quality management system, GMPs, risk and knowledge management, based on the PDCA (Plan-Do-Check-Act) cycle. It was concluded that knowledge management contributes with the proper functioning of quality management and GMPs; risk management is also a facilitating factor for the attainment of the organization objectives and quality, when it is focused on the most critical aspects of the organization and the manufacture processes, thus increasing safety of patients and reducing adverse reactions, complaints, deviations or withdrawal of products from the market.

Keywords: Quality management system, ISO 9001:2015, GMP, Risk management, Knowledge management, integrated systems.

\section{RESUMO}

Os laboratórios farmacêuticos na Colômbia devem cumprir as normas sanitárias, adotadas a partir de normas internacionais, como as BPF relacionadas à produção e controle de qualidade de medicamentos, no entanto, nessas organizações há pouca evidência de 
integração dos processos. Nessa perspectiva, o objetivo da pesquisa foi investigar a percepção dos funcionários sobre 0 atual gerenciamento dos sistemas de gestão nas organizações, as oportunidades de melhoria e os aspectos da gestão de riscos e do conhecimento que contribuem para a integração destas normas, visando construir uma proposta de integração. Por isso, foi efetuado um estudo combinado com alcance descritivo. Pesquisas semiestruturadas foram realizadas ao pessoal diretor e duas pesquisas, uma ao pessoal de nível tático e outra ao pessoal de nível operacional. Uma as conquistas foi a geração de uma proposta de integração do sistema de gestão da qualidade, as BPF, a gestão do risco e do conhecimento, baseada no PHVA. Conclui-se que gestão do conhecimento fornece uma contribuição no funcionamento certo da gestão da qualidade e BPF. Igualmente, a gestão do risco é um facilitador para 0 adimplemento dos objetivos organizacionais e de qualidade, Quando focado nos aspectos mais críticos da organização e dos processos de fabricação, aumentando dessa forma a segurança aos pacientes e reduzindo então as reações contrárias, reclamações, desvios ou retiradas de produto do mercado.

Palavras-chave: Sistema de gestão da qualidade, ISO 9001:2015, BPF, Gestão do risco, Gestão do conhecimento, Sistemas integrados.

\section{INTRODUCCIÓN}

La calidad y la industria farmacéutica han estado estrechamente relacionadas desde décadas atrás por su compromiso directo con la salud de los pacientes. Esto ha despertado conciencia en diferentes niveles no solo el organizacional, sino también el gubernamental, lo que la ha convertido en una de las industrias más reguladas a nivel mundial desde los años cincuenta (Haleem, Salem, Fatahallah y Abdelfattah, 2014).
Esta regulación está enfocada en el producto y los procesos que interfieren en su fabricación, por lo cual su inherencia en el crecimiento y desarrollo de la organización no se puede apreciar de una forma directa. Es recomendable la implementación de otras herramientas para el logro de los objetivos estratégicos, como la familia de las normas ISO 9000 de sistemas de gestión de calidad, y el apoyo de facilitadores, como la gestión del riesgo y la del conocimiento (García, Vallejo y Mora, 2015).

En el ámbito farmacéutico tanto la gestión del conocimiento como la del riesgo han sido la base de diferentes guías técnicas para el desarrollo del producto, el aseguramiento de la calidad y la productividad. Ambas han catalizado progresivamente la evolución de la industria farmacéutica desde la producción en masa poco flexible hacia la innovación en productos y procesos, evidenciada hoy por hoy en la solución de retos como la personalización de tratamientos farmacológicos, el control de procesos productivos en tiempo real y la demanda variable en el consumo de medicamentos (García et al., 2015).

Un ejemplo a nivel mundial es el caso de Italia, donde se implementó un sistema de calidad basado en los estándares de BPM (Buenas Prácticas de Manufactura) e ISO 9001 para la fabricación de radiofármacos inyectables en un centro de investigación público. Dicha integración permitió garantizar la calidad del producto final a través de las BPM y centrarse en otros aspectos no contemplados, como el diseño, la planificación, el análisis de datos y la revisión por la dirección, con un enfoque hacia la mejora continua, que ayuda al desarrollo general de la organización. Adicionalmente, se destaca que la conformación de esta integración promueve el uso de la gestión del riesgo en todas las etapas del ciclo de vida del producto (Poli, Petroni, Pardini, Salvadori y Menichetti, 2012).

Otro caso de integración se realizó en un centro de investigación y desarrollo farmacéutico ubicado en España. 
En la investigación realizada se implementó un SGC (sistema de gestión de calidad) incorporando la norma ISO 9001 a las BPM con el fin de buscar el reconocimiento de la excelencia a través del mejoramiento continuo y la alta productividad. Asimismo, se planteó que un programa de garantía de calidad individual cuando se manejan varios sistemas de gestión es muy difícil de manejar. Por eso se seleccionó el SGG de la norma ISO 9001, por su carácter de aplicación universal. Los logros con esta integración fueron beneficios como mejoras en la satisfacción de los clientes, mayor participación de la dirección, creación de un ambiente de compromiso y conciencia entre los empleados y una mejor asignación de recursos (Fábregas, García, Pérez, Suñé, Tico y Miñarro, 2010).

En los últimos años la gestión integral del riesgo ha cobrado importancia en la industria farmacéutica. Con las directrices de la ICH (2005) sobre gestión de riesgos de calidad se ha podido establecer el estándar del futuro en materia de riesgos para este tipo de industria (Guebitz, Schnedl y Khinast, 2012). Es asícomo la investigación realizada en Polonia, en donde se integró la gestión del riesgo basado en la norma ISO 31000 al SGC de la norma ISO 9001, se enfocó en una mayor protección de sus procesos y el cumplimiento cabal de sus objetivos. Para lo cual se encontró que los elementos de ambos sistemas se complementan y enriquecen, lo que aumenta la eficacia para la consecución de las metas de la organización (Golas, 2014).

La industria farmacéutica en Colombia, como en muchos países, se rige por reglamentaciones internacionales. En el país estas han sido acogidas por el Ministerio de Salud y de la Protección Social como las BPM, armonizadas y emitidas por la OMS (Organización Mundial de la Salud) en su Informe n. ${ }^{0} 32$ de 1987, de la Serie Informes Técnicos 823. Se busca asegurar la calidad de los medicamentos disminuyendo los riesgos existentes durante toda la cadena productiva y que no se pueden evitar con el control final de los productos (OMS, 2010).
Siendo la implementación de la norma ISO 9001 de carácter voluntario, no todos los laboratorios farmacéuticos optan por ella, y lo hacen solo por el cumplimiento de la reglamentación sanitaria. Esta puede ser una de las razones por las cuales no se encontraron investigaciones que se enfoquen en la integración de estos sistemas con la gestión del riesgo y la del conocimiento.

Es importante destacar la investigación realizada por María del Rosario Velázquez, titulada "Planificación de un sistema de gestión de la calidad como plataforma para integrar otros modelos de gestión", donde la autora establece una continuidad de su trabajo en el sentido de ahondar en la recomendación sobre "desarrollar una investigación que trabaje los elementos que van más allá de la planificación del SGC, es decir no contemplados, como la realización del producto en el tema de controles y mediciones" (2012, p. 138), aspecto fundamental contemplado en las BPM.

Específicamente en el sector farmacéutico existe una investigación que determinó y evaluó la gestión del conocimiento en una organización de este tipo, que encontró una alta necesidad de generación de conocimiento que ayude a solventar los problemas y emprender acciones para el mejoramiento de los procesos. Asimismo, tal investigación enfatiza en que la organización se concientice de la importancia, los beneficios y los impactos del conocimiento y su estrecha relación con el SGC (Babativa y Hernández, 2011).

Sin embargo, a pesar de manejar estos lineamientos de BPM y contar con sistemas de gestión de calidad basados en la norma ISO 9001, que por sí solos aportan valor a la organización, la industria farmacéutica está enfocada en la prevención y el control de rechazos, reprocesos, confusiones, productos no conformes, devoluciones, quejas y reclamos. Y, según lo exponen García et al. (2015), esta industria en Colombia se ha quedado corta en la 
implementación de otros estándares internacionales que la pongan en la vanguardia a nivel mundial y la saquen del estancamiento de la tasa de apertura exportadora en que se encuentra debido, entre otras razones, a la poca innovación amarrada a problemas tecnológicos y de bajo desarrollo del conocimiento, que dificultan asegurar la calidad de los productos actuales y desarrollar nuevos productos.

El resultado de estas reflexiones permite reconocer que la integración del SGC con las BPM, apoyado en la gestión del riesgo y la del conocimiento, es una herramienta que, al implementarse de una manera sistemática, puede aportar en el logro de la satisfacción de los pacientes y profesionales de la salud, al brindar un medicamento más efectivo, seguro y eficaz. Así como en la satisfacción de los clientes, al disminuir errores en el servicio de entrega del producto y mejorar tiempos de abastecimiento, entre otros. También redunda en la satisfacción de los miembros de la organización, al facilitar el desarrollo de sus procesos y gestión, y en la satisfacción de los accionistas, al garantizar más confianza en el crecimiento y sostenibilidad de la organización. Finalmente, las autoridades sanitarias de cualquier país tendrán más seguridad del cumplimiento de los requisitos legales sanitarios por parte de los laboratorios farmacéuticos nacionales, lo que facilita el ingreso de los medicamentos.

En este estudio se buscó indagar la percepción de los empleados sobre el manejo actual de los sistemas de gestión en las organizaciones, las oportunidades de mejora en el desempeño de estos sistemas y los aspectos de la gestión del riesgo y del conocimiento que aportan en la integración del SGC basado en la norma ISO 9001:2015 con las BPM. El objetivo fue construir una propuesta de dicha integración, que permita subsanar los inconvenientes detectados en las empresas y avanzar hacia estándares internacionales que brinden confianza a todas las partes interesadas de las compañías.

\section{METODOLOGÍA}

El enfoque de la investigación es mixto, para lo cual se definió utilizar el diseño de triangulación concurrente (Ditriac), mediante el cual

de manera simultánea se recolectan y analizan datos cuantitativos y cualitativos sobre el problema de investigación aproximadamente en el mismo tiempo. Durante la interpretación y la discusión se terminan de explicar las dos clases de resultados, y generalmente se efectúan comparaciones de las bases de datos. (Hernández, Fernández y Baptista, 2010, p. 613)[

Este diseño no tiene preferencia por ningún método. Ambos tienen el mismo peso en la investigación y se realizaron al tiempo en la medida en que cada empresa permitió desarrollarlos.

Para el desarrollo de la investigación se utilizaron dos instrumentos: en el método cualitativo, una entrevista semiestructurada al personal directivo, y en el método cuantitativo, dos encuestas, una al personal de nivel medio, que coordina y realiza seguimiento, y otra al personal de nivel bajo, que ejecuta la operación. Estos instrumentos se elaboraron teniendo en cuenta las variables y categorías establecidas para la investigación, definidas en el anexo A ("Operacionalización de la investigación”). Estas categorías surgen del análisis teórico realizado a los artículos encontrados a partir de la búsqueda bibliográfica enfocada en los sistemas de gestión involucrados en esta investigación: SGC, BPM, gestión del riesgo y gestión del conocimiento, en un rango de tiempo de cinco años, entre 2011 y 2015, en cuatro bases de datos: Science Direct, Redalyc, Scopus y Ebscohost.

Para garantizar que los instrumentos usados realmente midieran las variables que se pretendían evaluar, se sometieron a una validación de contenido que evaluó la suficiencia, claridad, coherencia y relevancia de los ítems. 
La confiabilidad de los dos instrumentos cuantitativos, entendida como la capacidad del instrumento de arrojar resultados veraces y constantes cuando es aplicado en repetidas ocasiones y bajo las mismas condiciones (Martín, 2004), se estimó con el coeficiente de alfa de Cronbach, como medida de coherencia o consistencia interna. El coeficiente se calculó a partir de las varianzas de los valores obtenidos de la calificación de cada evaluador. Los valores obtenidos permitieron establecer que los ítems de ambos instrumentos están estrechamente relacionados con las variables que se midieron. Por lo tanto, cada instrumento tiene una alta confiabilidad.

La población se delimitó así: empresas ubicadas en la ciudad de Bogotá, que fabriquen medicamentos inyectables. Esto corresponde a un total de catorce empresas, de acuerdo con lo confirmado por el Invima (Instituto Nacional de Vigilancia de Medicamentos y Alimentos. Sev definió realizar el muestreo por conveniencia debido a la dificultad de acceso a las empresas, es decir, a aquellas empresas que aceptaran participar en el estudio después de hacer llegar una invitación a todas. Finalmente aceptaron participar tres empresas. Como este número no es representativo, no se puede generalizar o hacer inferencia de los resultados obtenidos a toda la población, a partir de la aplicación de los instrumentos en las tres empresas que accedieron a participar en el estudio.

Es así que, debido a las circunstancias, el estudio no podría clasificarse como probabilístico. Los resultados solo pueden extenderse a empresas con las mismas características de las empresas participantes. Para efectos de mantener la confidencialidad de dichas empresas, se asignó un código a cada una. Las siguientes son las características que las distinguen:

- Empresa farmacéutica A: fabricante de medicamentos únicamente inyectables para uso humano, con una trayectoria de dieciocho años en el mercado y 67 empleados. Clasificada como mediana empresa de acuerdo con el valor de sus activos totales. Es de carácter familiar, con capital nacional y ventas a nivel nacional e internacional. Se encuentra certificada en BPM, tiene implementado el SGC basado en la norma ISO 9001:2008 sin certificar y no cuenta con ningún otro sistema de gestión.

- Empresa farmacéutica B: fabricante de medicamentos únicamente inyectables para uso humano, trayectoria de tres años en el mercado, cuenta con veintisiete empleados y es clasificada como mediana empresa de acuerdo con el valor de sus activos totales. Es de carácter familiar, con capital nacional y ventas solo a nivel nacional. Se encuentra certificada en BPM, tiene implementado el SGC basado en la norma ISO 9001:2008 sin certificar y no cuenta con ningún otro sistema de gestión.

- Empresa farmacéutica C: fabricante de medicamentos inyectables y no inyectables para uso humano, trayectoria de 45 años en el mercado, con 39 empleados. Está clasificada como mediana empresa de acuerdo con el valor de sus activos totales. Es de carácter familiar, de capital nacional y ventas solamente a nivel nacional. Se encuentra certificada en BPM, tiene implementado el SGC basado en la norma ISO 9001:2008 sin certificar y no cuenta con ningún otro sistema de gestión.

Para el análisis de los datos cuantitativos se tabularon primero los datos recabados de las tres empresas en una matriz de Excel y posteriormente, con la ayuda de Excel, se elaboró un análisis descriptivo de distribución de frecuencias de los datos por cada una de las preguntas, que relacionó las variables y el compilado del estadístico descriptivo. El procesamiento 
de la información cualitativa se realizó con ayuda del programa Atlas. Tiß日, a través de la codificación abierta y axial. Un segundo paso fue comparar las categorías para interpretar la información, encontrar patrones repetitivos o contrarios que permitieron generar explicaciones y formar estructuras (mapas conceptuales y redes semánticas) y temas que fueron la base para la generación de las conclusiones. Finalmente, se triangularon los análisis de ambos métodos, comparando e interpretando los resultados, lo que permitió el diseño del resultado final, la propuesta de integración.
RESULTADOS Y DISCUSIÓN

\section{Resultados y análisis de la información cuantitativa}

\begin{abstract}
Después de tabular la información recabada de las 52 encuestas aplicadas a los mandos medios y las 108 encuestas aplicadas al personal operativo de las tres empresas farmacéuticas de inyectables, se realizó un análisis descriptivo de distribución de frecuencias de los resultados por pregunta, que se encuentra resumido en las tablas 1 y 2 .
\end{abstract}

Tabla 1. Resumen resultados encuestas mandos medios

\begin{tabular}{|c|c|}
\hline Pregunta & Resultado \\
\hline 1. ¿Se tiene implementado un SGC en la organización? si la respuesta es afirmativa, señale la norma bajo la cual está implementado & 67 \\
\hline 2. ¿En qué número de informe técnico de la OMS se basan las BPM que tienen actualmente implementadas? & 79 \\
\hline $\begin{array}{l}\text { 3. ¿Cuentan con alguna metodología o modelo para gestionar el riesgo (identificación, análisis, evaluación y tratamiento)? si la } \\
\text { respuesta es afirmativa, señale el modelo bajo el cual está implementado }\end{array}$ & 38 \\
\hline $\begin{array}{l}\text { 4. ¿Se sigue una herramienta de gestión para administrar el conocimiento organizacional? si la respuesta es afirmativa, señale el } \\
\text { modelo bajo el cual está implementada }\end{array}$ & 4 \\
\hline $\begin{array}{l}\text { 5. EEstá integrado el SGC con algún otro sistema o herramienta de gestión en la organización? si la respuesta es afirmativa, señale el } \\
\text { sistema o herramienta }\end{array}$ & 69 \\
\hline 6. El SGC involucra todas las áreas de la organización & 90 \\
\hline 7. Los objetivos del SGC están articulados con las metas, objetivos y estrategias de la organización & 96 \\
\hline $\begin{array}{l}\text { 8. Al hacer seguimiento del desempeño de la organización, la dirección de la empresa tiene en cuenta información proveniente del } \\
\text { SGC y de las BPM }\end{array}$ & 82 \\
\hline 9. La integración de las BPM con el SGC ayuda al cumplimiento de los objetivos, metas y estrategias de la organización & 96 \\
\hline 10. En el SGC se incluyen todos los aspectos de las BPM & 88 \\
\hline 11. Las BPM y el SGC tienen un enfoque preventivo & 81 \\
\hline 12. El programa de auditorías está basado en un análisis de riesgos & 69 \\
\hline 13. El cumplimiento de las BPM es desarrollado de una manera natural por todos los que intervienen en ello & 75 \\
\hline $\begin{array}{l}\text { 14. El desarrollo de nuevos medicamentos, procesos o servicios es el resultado del flujo continuo de información y conocimiento en } \\
\text { toda la organización }\end{array}$ & 67 \\
\hline 15. Los objetivos de las BPM están articulados con las metas, los objetivos y las estrategias de la organización & 92 \\
\hline 16. En la organización las BPM abarcan desde el diseño de los medicamentos hasta la administración al paciente & 77 \\
\hline 17. Las BPM generan confianza en la organización sobre la calidad de los medicamentos & 98 \\
\hline $\begin{array}{l}\text { 18. Se puede mejorar la calidad de los medicamentos, procesos y servicios de la organización gestionando los riesgos y el } \\
\text { conocimiento organizacional }\end{array}$ & 98 \\
\hline 19. El nivel directivo muestra un compromiso permanente con el cumplimiento de las BPM & 87 \\
\hline $\begin{array}{l}\text { 20. Los riesgos inherentes a la fabricación y el control de medicamentos se analizan y evalúan pensando en la protección del } \\
\text { paciente }\end{array}$ & 87 \\
\hline 21. Los riesgos inherentes a las actividades de BPM se evalúan de manera técnica, es decir, no suponen una visión estratégica & 54 \\
\hline
\end{tabular}




\begin{tabular}{|l|c|}
\hline \multicolumn{1}{|c|}{ Pregunta } & Resultado \\
\hline 22. La gestión del riesgo involucra todos los procesos de la organización & 83 \\
\hline $\begin{array}{l}\text { 23. Cuando se toman decisiones en los distintos niveles de la organización, se tienen en cuenta los riesgos que puedan influir en los } \\
\text { resultados que se quieren lograr }\end{array}$ & 64 \\
\hline 24. Se divulgan los riesgos a los cuales están expuestos los trabajadores, la organización y el medio ambiente & 79 \\
\hline $\begin{array}{l}\text { 25. La planificación de la organización se realiza teniendo en cuenta los resultados del análisis de riesgos y toda la información } \\
\text { relevante }\end{array}$ & 65 \\
\hline $\begin{array}{l}\text { 26. Cuando se definen los objetivos estratégicos de la organización, se tienen en cuenta aprendizajes obtenidos a partir de los logros } \\
\text { y las dificultades que se han presentado }\end{array}$ & 73 \\
\hline $\begin{array}{l}\text { 27. Los resultados que ha obtenido la organización se deben al enfoque en el conocimiento organizacional } \\
\text { 28. La información necesaria para garantizar el cumplimiento de objetivos y metas de la organización se encuentra siempre } \\
\text { disponible para quien la requiere }\end{array}$ & 79 \\
\hline 29. A través de su conocimiento, los colaboradores de la organización son los generadores de valor para esta & 69 \\
\hline 30. La autoridad de los cargos en la organización está estrechamente relacionada con el nivel de conocimiento & 89 \\
\hline 31. La selección y promoción de cargos en la organización está basada en las competencias y los talentos de las personas & 79 \\
\hline 32. Las políticas de comunicación están basadas en el conocimiento que se tiene de la organización y de los clientes & 79 \\
\hline 33. En la organización se invierten el tiempo y los recursos suficientes en la capacitación y formación de todos los colaboradores & 71 \\
\hline 34. El conocimiento y aprendizaje del personal es una de las principales inquietudes de la dirección de la organización & 56 \\
\hline
\end{tabular}

Fuente: elaboración propia.

Tabla 2. Resumen resultados encuestas al personal operativo

\begin{tabular}{|c|c|}
\hline Pregunta & Resultado \\
\hline 1. Tengo claro para qué se implementó el SGC & 88 \\
\hline 2. El SGC involucra todas las áreas de la organización & 88 \\
\hline 3. Se realiza divulgación de las metas, los objetivos y las estrategias por parte del nivel directivo a toda la organización & 66 \\
\hline 4. Periódicamente se informa sobre los resultados de los objetivos y metas del SGC & 65 \\
\hline 5. Percibo que toda la organización está comprometida en la implementación y desarrollo del SGC & 70 \\
\hline 6. Percibo que toda la organización está comprometida en la implementación y el desarrollo de las BPM & 68 \\
\hline 7. Las BPM y el SGC controlan lo mismo & 72 \\
\hline 8. El cumplimiento de las BPM es desarrollado de una manera normal por todos los que intervienen en la organización & 63 \\
\hline 9. Mis ideas y conocimientos son tenidos en cuenta para el desarrollo de nuevos medicamentos, procesos o servicios & 28 \\
\hline 10. Las BPM generan confianza dentro de la organización sobre la calidad de los medicamentos & 90 \\
\hline 11. El nivel directivo muestra compromiso con el cumplimiento de las BPM & 82 \\
\hline 12. Estoy capacitado en el SGC & 60 \\
\hline 13. Estoy capacitado en las BPM & 77 \\
\hline 14. Se analizan los riesgos en la fabricación de los medicamentos teniendo en cuenta la protección del paciente & 83 \\
\hline 15. Las decisiones relacionadas con la fabricación de productos se toman teniendo en cuenta los riesgos asociados & 75 \\
\hline 16. Conozco los riesgos a los cuales estoy expuesto en el trabajo & 89 \\
\hline 17. Estoy capacitado en la prevención de riesgos & 73 \\
\hline 18. La organización aprende a partir de los errores que se cometen & 73 \\
\hline 19. La organización aprende de sus logros & 74 \\
\hline $\begin{array}{l}\text { 20. La información necesaria para garantizar el cumplimiento de objetivos y metas de la organización se encuentra al alcance de } \\
\text { quien la requiere }\end{array}$ & 69 \\
\hline
\end{tabular}


Integración de la gestión de la calidad con las buenas prácticas de manufactura en tres empresas farmacéuticas de inyectables en Bogotá, D. C

\begin{tabular}{|l|c|}
\hline \multicolumn{1}{|c|}{ Pregunta } & Resultado \\
\hline 21. La empresa reconoce que son los colaboradores los que logran los resultados y beneficios para la organización & 66 \\
\hline 22. La autoridad de los cargos en la organización está estrechamente relacionada con el nivel de conocimiento & 74 \\
\hline 23. La selección y promoción de cargos en la organización está basada en las competencias y los talentos de las personas & 59 \\
\hline 24. En la organización se invierten el tiempo y los recursos suficientes en la capacitación y formación de todos los colaboradores & 68 \\
\hline 25. El conocimiento es una herramienta importante dentro de la organización & 91 \\
\hline
\end{tabular}

Fuente: elaboración propia.

Del análisis de la información anterior surgen los productos que dan respuesta al primer objetivo específico de la investigación:

\section{Diagnóstico en empresas farmacéuticas de inyectables sobre la percepción de los empleados hacia los SGC, las BPM, la gestión de los riesgos y la del conocimiento y su articulación en las organizaciones}

De acuerdo a los resultados expuestos, para los los mandos medios, quienes coordinan y realizan el seguimiento en sus organizaciones, no existen metodologías o herramientas formales que les permitan administrar los riesgos y gestionar el conocimiento, aunque esto se realiza de manera empírica pero no sistemática. En efecto, a preguntas relacionadas con el manejo de los riesgos — por ejemplo, si tienen en cuenta el riesgo en la planificación de la gestión de la organización o si se basan en el estudio de riesgos para la toma de decisiones- las respuestas estuvieron en un rango de puntuación media. Y las respuestas a preguntas con un enfoque del conocimiento - por ejemplo, si la información está disponible o el desarrollo de nuevos productos o servicios es el resultado del flujo continuo de información y conocimientos- se mantuvieron en ese mismo rango medio, lo que denota poca fuerza en estas afirmaciones. Asimismo, en un rango más bajo estuvieron afirmaciones sobre la posición en que se encuentra el conocimiento en las organizaciones y la mirada estratégica que les aporta gestionar los riesgos.
El hecho de que solo el $67 \%$ de los participantes responda positivamente sobre si está implementado el SGC, denota que el manejo y difusión del sistema requiere ser fortalecido. Y que un $69 \%$ afirme que el SGC está integrado con otros sistemas de gestión o herramientas, sin estarlo, reafirma la falta de conocimiento en integración de sistemas de gestión o la necesidad de que la forma de gestión en las organizaciones sea tal que se trabaje de manera interconectada en los diferentes aspectos ambientales, de calidad, de BPM y de seguridad y salud en el trabajo.

Puntuaciones altas (90-100\%) para afirmaciones sobre si los objetivos del SGC y de las BPM están alineados con los objetivos y metas de la organización y si las BPM generan confianza sobre la calidad de los medicamentos, permiten percibir que las organizaciones están enfocadas en el cumplimiento de estándares normativos de calidad de medicamentos y calidad del servicio. Es importante destacar que estas puntuaciones altas también fueron para afirmaciones enfocadas en que se puede mejorar la calidad de los productos y servicios gestionando los riesgos y el conocimiento. Esto afirma la relevancia para los participantes de esas dos herramientas de gestión en las organizaciones. Asimismo, que se haya respondido con un $96 \%$ de favorabilidad a la afirmación de que la integración del SGC y las BPM ayuda al cumplimiento de los objetivos y metas de la organización reafirma la importancia que tiene en esta el enfoque de la calidad en todos los aspectos. 
Los resultados encontrados permiten apreciar los aspectos que percibe el personal que realiza las operaciones, mandos bajos en las organizaciones. En primer lugar, la percepción menos favorable (28\%) es para la afirmación sobre si sus ideas y conocimientos son tenidos en cuenta para el desarrollo de nuevos medicamentos, productos o servicios, lo que permite ver que la organización es débil en escuchar a todos los colaboradores o que no cuenta con herramientas que le permitan materializar los conocimientos adquiridos por las personas que realizan la operación. Entre 50-60\% de percepción favorable se ubicaron afirmaciones como "Estoy capacitado en el SGC" y "La selección y promoción de cargos está basada en las competencias de las personas", lo que permite inferir que el conocimiento no es un concepto fuerte en las organizaciones ni el aprendizaje es prioritario en cada empleado, y además, que no se ven las competencias como una herramienta para medir y evaluar el desarrollo de cada cargo. Una de las puntuaciones más altas es contradictoria con esta última apreciación, pues el 91\% afirma que el conocimiento es una herramienta importante dentro de la organización. Ello puede indicar que el conocimiento se percibe como importante conceptualmente, pero que no se cuenta con los medios adecuados para gestionarlo.

Otra afirmación favorablemente positiva es que las BPM generan confianza sobre la calidad de los medicamentos $(90 \%)$. Esto indica la importancia que los participantes le dan a dicha herramienta de gestión por encima del SGC. Por ello es importante aprovechar esta cultura de calidad de los procesos productivos para permear el resto de los procesos de la organización. Puntuaciones entre el 60-70\% en afirmaciones referentes a la divulgación periódica de resultados por parte del nivel directivo a toda la organización, el compromiso general hacia la implementación y desarrollo de los sistemas de gestión, el cumplimiento de las BPM desarrollado de una manera habitual, la disponibilidad de la información para el logro de objetivos, el reconocimiento de que son los colaboradores los que logran los resultados y la inversión de tiempo y recursos suficientes en la capacitación y formación de sus empleados, denotan que las empresas necesitan involucrar en su gestión aspectos fundamentales para lograr un resultado eficaz de sus objetivos, como la comunicación, el compromiso, la toma de conciencia, la disponibilidad de la información, el reconocimiento a los empleados y la formación del talento humano. Estos aspectos están inmersos implícitamente en los requerimientos de las BPM, la norma ISO 9001:2015 y las herramientas de la gestión del riesgo y del conocimiento.

\section{Resultados y análisis de la información cualitativa}

Después de transcribir y analizar las ocho entrevistas realizadas a los directivos de las tres empresas farmacéuticas, surgen los productos que dan respuesta a los objetivos específicos segundo y tercero.

\section{Oportunidades de mejora en el desempeño de los sistemas de gestión que tienen las empresas farmacéuticas de inyectables entrevistadas}

Una oportunidad para este tipo de empresas es entender que los sistemas de gestión están diseñados para ayudar a las organizaciones a cumplir sus objetivos y que su implementación o certificación no es un objetivo en símismo. En ese sentido es importante que la alta dirección se involucre y comprometa en apoyar su implementación y desarrollo más allá del tema económico, y se convierta en el ente articulador y motivador de todos los procesos. Que difunda sus objetivos y metas con toda la organización y promueva la articulación con los diferentes sistemas de gestión. El enfoque preventivo es un aspecto aún poco explorado en estas empresas, pero de gran importancia en los procesos productivos por la criticidad de sus productos. Se requiere la implementación de un análisis sistemático y continuo de los riesgos, que involucre la 
participación del personal directamente implicado. La reactividad es un aspecto predominante en el día a día, así que es indispensable que se migre a un análisis más preventivo de todos los aspectos que puedan impactar la calidad de los medicamentos y sus procesos. Y hay que dejar de ver los requerimientos normativos (cuarentena de productos, validaciones y calibraciones de equipos, capacitaciones al personal, auditorías, entre otros) como los únicos aspectos preventivos a tener en cuenta. Todo lo anterior es derivado del desconocimiento que se tiene de las herramientas existentes para la gestión del riesgo. $\mathrm{Al}$ ser estas empresas tan reguladas y vigiladas por las entidades sanitarias en sus aspectos técnicos y normativos, es importante que el conocimiento sea un pilar en el mantenimiento de sus procesos y en el desarrollo de sus productos, de modo que pueda ser transferido fácilmente y esté al alcance de quien lo requiera para el cumplimiento de objetivos. Se debe avanzar en entender que el conocimiento no está plasmado únicamente en los documentos, sino también en la forma de operar diariamente de cada miembro de la organización. Y se debe comprender que las capacitaciones como se están realizando no garantizan una difusión e interiorización de este conocimiento.

Muy de la mano del conocimiento está el desarrollo de competencias en el personal, un aspecto que permitirá a estas empresas avanzar hacia los estándares de calidad que se están imponiendo a nivel mundial en temas de buenas prácticas de manufactura farmacéutica y desarrollo de productos y servicios. Una oportunidad de mejora para este tipo de empresas es poder atraer y retener el talento humano brindando planes de carrera acordes a sus necesidades e intereses. Algo que en muchos casos se logra con la motivación del aprendizaje que pueda obtener el colaborador en el desarrollo de sus funciones.

Una oportunidad para el aprendizaje organizacional es que los aciertos o desaciertos que tenga la empresa puedan ser difundidos hacia una buena parte de la organización, y que esta los pueda entender y analizar, de manera que se genere un conocimiento a partir de allí.

\section{Aspectos de la gestión del riesgo y la gestión del conocimiento que aportan en la articulación de la integración del SGC y las BPM}

El diseño y desarrollo de medicamentos en estas empresas, que es un tópico de las BPM, requiere un conocimiento técnico profundo de los procesos de fabricación y de los compuestos químicos a formular. Asimismo, en el SGC el diseño y desarrollo de productos y servicios tiene un componente alto en cuanto a conocimiento de la naturaleza de los productos y de los requisitos funcionales y legales, entre otros. La cultura de la calidad en las empresas se alimenta del conocimiento que cada persona tiene frente a los requerimientos técnicos de las BPM o la gestión de los sistemas de calidad. Es así como el conocimiento se torna un elemento en común en ambos sistemas. Los hábitos de comportamiento en las áreas de fabricación de medicamentos inyectables son cruciales para obtener los resultados esperados. Los riesgos en estas empresas son percibidos como sucesos que ya pasaron y no son fundamentales para la toma de decisiones, pero uno de los principios de las normas ISO 9001, es la toma de decisiones basada en un análisis de la información y, por supuesto, de los posibles riesgos. El enfoque de las BPM actualmente también se basa en evaluar todas las posibles afectaciones en el producto, que puedan ser evitadas antes de llegar a ocurrir. Con ambos sistemas, BPM e ISO 9001, se busca la mejora de los procesos como ideal de las empresas. Es allí donde gestionar los riesgos aporta no solo a prevenir, sino también a potencializar los efectos deseables de cualquier riesgo, algo que las empresas en estudio no tienen a su favor. La gestión del riesgo y la del conocimiento van a dinamizar la implementación y el desarrollo de los sistemas integrados de gestión pues ambas requieren del involucramiento de gran parte del personal y de diferentes 
procesos y se basan en la mejor información disponible y en necesidades o requisitos previamente establecidos.

\section{Propuesta para la articulación de un} sistema integrado de gestión de la calidad con las BPM, apoyado en la gestión del riesgo y la del conocimiento para empresas farmacéuticas de inyectables

Al comparar la información obtenida por ambos métodos, se observan similitudes en las respuestas en aspectos como la falta de herramientas o metodologías para gestionar el riesgo y el conocimiento, y por lo tanto, el desconocimiento de estas herramientas de gestión. También se piensa equivocadamente que se gestionan los riesgos al ejecutar o implementar actividades como consecuencia del cumplimiento de algún requisito normativo. El conocimiento se percibe por todos como lo que está descrito en los documentos, procedimientos 0 instructivos, como si esa fuera la única forma conocible para almacenarlo. Aunque se perciben más oportunidades de mejora y capacidades de detección de debilidades en las entrevistas realizadas a los directivos, en ambas partes se perciben falencias a nivel de la difusión y el procesamiento de la información. Tal parece que no se tiene en cuenta la experiencia de todas las personas en la organización para la resolución de problemas o el desarrollo de productos o servicios. Lo anterior indica una validez de criterio en el estudio pues la información no discrepa sustancialmente de un método a otro y permite confirmar o completar la información de ambos. Para formular la propuesta fue importante comparar los principios y requisitos de cada sistema o herramienta de gestión, en búsqueda de elementos comunes y no comunes que permitieran una correcta interacción.

Para estructurar la gestión del conocimiento la propuesta está basada en la publicación de Naciones Unidas "Introducción a la gestión del conocimiento y su aplicación al sector público" (Peluffo y Catalan, 2002). Y para la gestión del riesgo se usó la norma ISO 31000, "Gestión del riesgo. Principios y directrices".

Inicialmente se compararon los principios tomando como base los siete principios de la norma ISO 9001:2015 y evidenciando que las BPM, de los tres conjuntos normativos, son la que menos coincidencia tienen ya que no incluyen ninguno de los siguientes principios: enfoque al cliente, liderazgo, compromiso de las personas, enfoque a procesos y gestión de las relaciones. Y la herramienta de gestión del riesgo solamente deja de incluir el principio de liderazgo. Adicionalmente, las BPM incorporan como principios tres elementos que desde el punto de vista de los sistemas de gestión se clasificarían como requisitos operativos de la producción, a saber: 1) las responsabilidades gerenciales deben estar claramente especificadas en las descripciones de trabajo; 2) el producto debe ser acabado, procesado y controlado correctamente y de acuerdo con los procedimientos definidos; 3 ) se deben tomar medidas adecuadas para asegurar que los productos farmacéuticos sean almacenados, distribuidos y subsiguientemente manejados de forma que la calidad se mantenga durante todo su periodo de actividad.

Se elaboró, entonces, una tabla de correspondencias de los requisitos específicos teniendo como base la norma ISO 9001:2015 y clasificándolos de acuerdo a la metodología del ciclo PHVA (planear, hacer, verificar, actuar). De ella se destaca la falencia que tiene la norma de BPM frente a aspectos como evaluación del contexto de la organización, liderazgo, planificación y mejora. Sin embargo, tal norma tiene una total correspondencia frente a la operación (anexo 2, "Tabla de correspondencias de requisitos de cada sistema o herramienta de gestión, basada en el ciclo PHV”").

Con base en la información recabada y analizada a través de los instrumentos cuantitativos y cualitativos y en la correspondencia de principios y requisitos, se propone 
la siguiente metodología (figura 1) para la articulación siguiendo el ciclo PHVA.

\section{Planear}

1) Elaborar un diagnóstico inicial para determinar el estado actual de la organización en aspectos específicamente relacionados con la gestión del riesgo y del conocimiento puesto que los sistemas de gestión de la calidad y BPM ya se encuentran implementados. Es importante en esta etapa involucrar herramientas que midan el nivel de madurez de la organización en gestión del conocimiento para poder definir los recursos necesarios para su implementación y un diagnóstico de prácticas habituales.

2) Determinar los objetivos a lograr con el sistema integrado de gestión.

3) Incorporar el entendimiento del contexto de la organización, las necesidades y las expectativas de las partes interesadas que involucra la nueva versión de la norma ISO 9001:2015. Asimismo, considerar el contexto organizacional en el cual se van a gestionar el riesgo y el conocimiento, sus objetivos, su alcance y los recursos necesarios. También se debe contemplar el contexto legal, económico, cultural y tecnológico para el desarrollo de las BPM.

4) Establecer los roles y responsabilidades dentro del sistema integrado de gestión evitando solapamiento de autoridades. Esto es indispensable en las BPM puesto que, al ser un requerimiento legal, las responsabilidades y autoridades están reglamentadas para algunos cargos.

5) Establecer el mecanismo para planificar los cambios que se requieran del sistema integrado.
6) Definir los recursos necesarios para la implementación: capital humano, tecnologías, comunicación, información documentada, infraestructura física y de equipos, conocimiento organizacional.

7) Desde esta etapa se debe incorporar la valoración de los riesgos. Primero los asociados a la implementación del sistema integrado de gestión, que van a depender del contexto de cada organización. Previamente hay que definir los criterios que se van a utilizar para evaluar la importancia del riesgo: naturaleza y tipo de causas y consecuencias, método para medir la probabilidad, método para determinar el nivel de riesgo, nivel de riesgo aceptable.

8) Establecer el nivel de madurez de la gestión por procesos pues una debilidad de las empresas del estudio es la poca interacción entre los procesos actuales. Definir acciones para avanzar al siguiente nivel.

9) Definir una interrelación de los procesos o los nuevos procesos del sistema integrado (mapa de procesos).

10) Diseñar listas de verificación para establecer el grado de cumplimiento de cada requisito de las normas de BPM y del SGC. Así como establecer los requisitos legales y normativos a que están expuestos todos los procesos del sistema.

11) Determinar los tiempos requeridos para la implementación en el hacer, verificar y actuar.

12) Todo el desarrollo de la planificación y sus conclusiones deben incorporarse en un manual de calidad 0 un documento afín. 
Hacer

1) Sensibilizar a toda la organización en la importancia y los beneficios del sistema integrado de gestión y en la toma de conciencia del compromiso de cada uno para el logro de los objetivos organizacionales.

2) Implementar mecanismos para la incorporación de los principios del SGC y los de las BPM que no están inmersos en aquellos.

3) Establecer los cronogramas de trabajo para la implementación de los requisitos normativos a partir de la aplicación de las listas de verificación diseñadas.

4) Fortalecer el enfoque por procesos con la caracterización de cada proceso definiendo fuentes de entradas, actividades, entradas, salidas, receptores de salidas, controles y puntos de control, riesgos asociados, responsables e información documentada necesaria.

5) Establecer un plan de desarrollo de las competencias requeridas para la implementación.

6) De acuerdo con el nivel de madurez establecido para gestionar el conocimiento, implementar herramientas para su producción, almacenamiento, actualización, circulación y utilización.

7) Definir metas, estrategias e indicadores para el logro de los objetivos propuestos para el sistema de gestión integrado.

8) Elaborar el organigrama de la empresa, donde se observen claramente las líneas de autoridad y comunicación.

9) Diseñar cada uno de los procesos del sistema de gestión integrado sobre la base del mejor conocimiento de cada uno, involucrando a las personas que van a desarrollarlos. Si es necesario, con el acompañamiento de expertos técnicos.

10) Diseñar un mecanismo para planificar y gestionar los cambios del sistema.

11) Establecer los mecanismos de comunicación interna y externa.

12) Diseñar el sistema de manejo de la información documentada.

13) Continuar con la valoración de los riesgos y su tratamiento priorizando a través de una matriz los procesos y actividades más críticas.

14) Continuar alimentando el manual de calidad con la información que se va generando en esta etapa.

\section{Verificar}

1) Determinar los métodos y frecuencias de seguimiento, medición, análisis y evaluación que se requieran para obtener los resultados esperados de los procesos y lograr la eficacia del sistema de gestión integrado.

2) Definir el programa de autoinspecciones y auditorías internas y a proveedores, de acuerdo con los lineamientos de las BPM y la norma ISO 9001:2015, y establecer prioridades basadas en la evaluación de riesgos.

3) Realizar auditorías de seguimiento al cumplimiento del plan de implementación para garantizar su total ejecución. 
4) Establecer el mecanismo de revisión por la dirección incluyendo los aspectos relacionados con las BPM y la gestión del riesgo y del conocimiento.

\section{Actuar}

1) Establecer herramientas para el manejo de inconformidades y sus acciones correctivas de acuerdo con lo establecido en la norma ISO 9001:2015.
2) De acuerdo con la valoración y el tratamiento de los riesgos establecidos, generar herramientas para el manejo de las acciones preventivas que surjan del análisis.

3) Establecer cómo será el manejo de las oportunidades de mejora que surjan de la revisión por la dirección, de las autoinspecciones y auditorias y de la gestión del riesgo.

Figura 1. Mapa de procesos sugerido para el sistema de gestión integrado

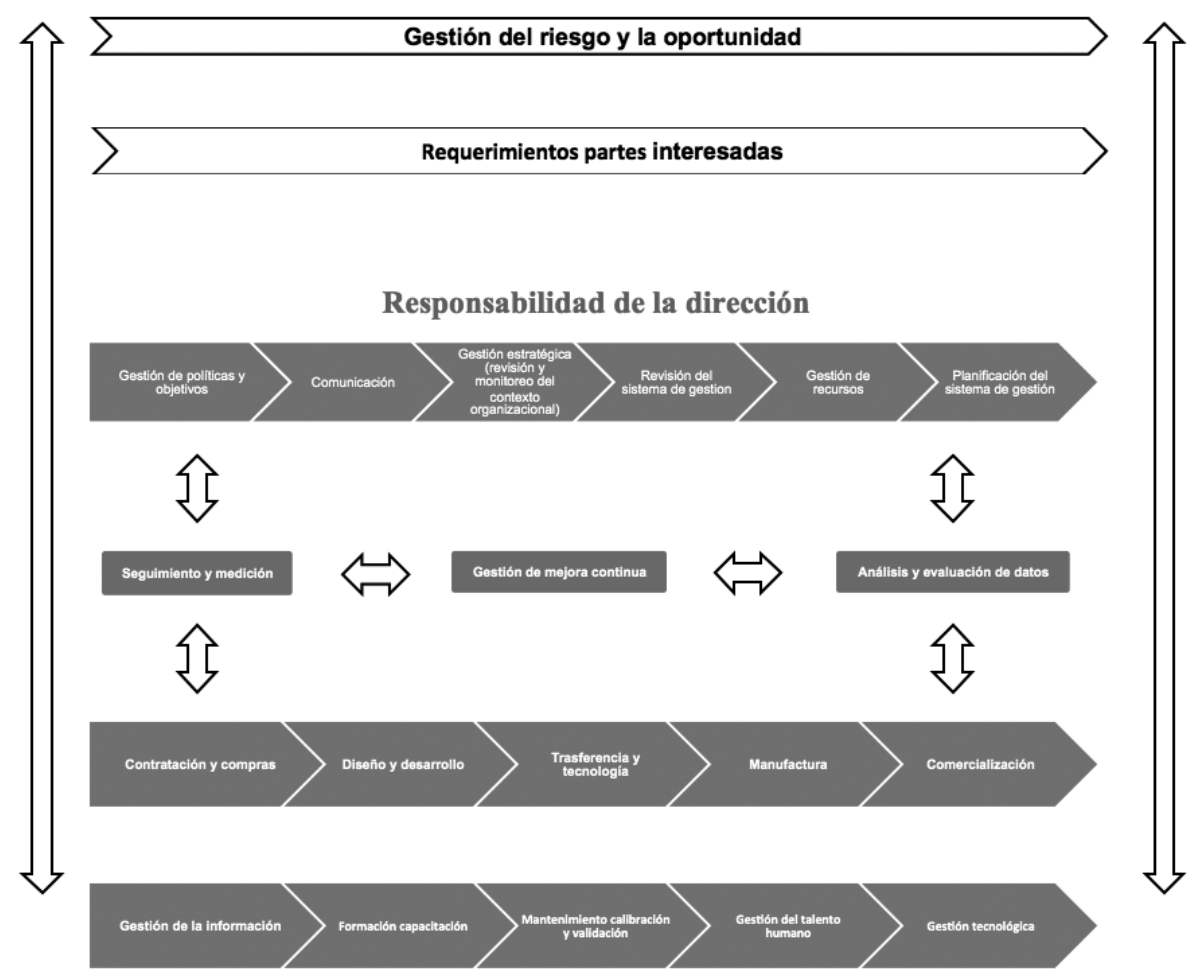

Fuente: elaboración propia.

\section{CONCLUSIONES}

A continuación se presentan las conclusiones de esta investigación en términos de los objetivos planteados.

Se observa que las organizaciones no están enfocadas en el análisis del riesgo, por la cultura que se tiene de mero cumplimiento de requerimientos y de creer que los errores 0 inconformidades son situaciones únicamente negativas y no una oportunidad para mejorar. En ese sentido, estudios como los desarrollados por Kawai, Kitajima, Fuchino, Sekia y Naka (2013) establecen 
la importancia de la evaluación de la calidad desde el diseño del producto y la utilidad de ver los errores y posibles errores como oportunidades para el aprendizaje a través del análisis de los diferentes escenarios en que las desviaciones afectan la calidad de sus procesos. Al final la organización obtiene un mejor entendimiento de sus actividades a través de un intercambio de información sobre todos los posibles riesgos. Un análisis de riesgos sistemático es un proceso que inicialmente puede resultar desgastante para las empresas, y por dicha inversión muchas no lo realizan, pero una vez estandarizado es una herramienta útil para la operación y seguridad de los procesos y productos, que finalmente puede ser cuantificada en términos de disminución de costos, errores y reprocesos, y de un mejor aprovechamiento de oportunidades (Kawai et al., 2013).

Otro aspecto importante en la gestión de estas empresas es que el conocimiento es reconocido verbalmente como indispensable para el desarrollo de la organización, pero en términos prácticos se desconoce la forma de gestionarlo. Las personas sienten que sus ideas y conocimientos no son tenidos en cuenta en el desarrollo de procesos o productos, pero también se observa que muchos no están interesados en el intercambio de información o no es fácil que lo hagan, bien sea por el temor a perder en cierta forma el control de una operación o bien porque no se cuenta con herramientas que permitan su captura de una manera adecuada. El cambio de la cultura de una organización en relación con que las personas decidan participar activamente en el intercambio de la información y el desarrollo de los procesos está asociado a que existan sistemas de gestión, como por ejemplo el de la calidad total, que concientizan a las personas sobre las ventajas de la diseminación del conocimiento y ayudan a transformar la información de tácita a explícita, lo que facilita el intercambio de conocimientos (Duran, Çetindere, y Şahan, 2014).
Otros estudios supeditan el éxito de los sistemas de gestión a la implementación de los cuatro aspectos principales de la gestión del conocimiento, a saber: creación, almacenamiento, transferencia y aplicación. En tal sentido, la gestión del conocimiento se refiere a un enfoque multidisciplinario para lograr los objetivos de la organización haciendo el mejor uso del conocimiento. Esto permite reconocer, de acuerdo a la información recabada sobre las empresas, que el conocimiento es un facilitador para la operación de los sistemas de gestión y que, por su relevancia tanto en las BPM como en el SGC, también ayudará a la integración de ellos (Kahreh, Shirmohammadi y Kahreh, 2014).

Los resultados de este estudio están en concordancia con lo expuesto por De la Hoz, Carrillo y Gómez (2012), quienes concluyen que la gestión del conocimiento ayuda a superar las dificultades que impiden el éxito de la implementación de la gestión de la calidad a través de diferentes herramientas que promueven el trabajo en equipo, entendido como compañerismo, por medio de la solución de problemas en diferentes áreas. La idea es aprovechar que en las empresas existe el interés de las personas de aportar al desarrollo de la organización, sentirse útiles y ser reconocidas por sus compañeros; de realizar su trabajo de manera que contribuya con el logro de los objetivos esperados. Pero esto no se logra si la empresa no brinda herramientas y ambientes adecuados, que ayuden a sus colaboradores a poner todas sus capacidades al servicio de los objetivos organizacionales (De la Hoz et al., 2012). Es allí donde la gestión del conocimiento — entendida no como la forma de organizar y controlar documentos o información, sino como el soporte para capturar el conocimiento, difundirlo, aplicarlo en favor del mejoramiento de los procesos, diseño y desarrollo de nuevos productos y facilitar así la formación de una cultura organizacional enfocada en la calidadaporta tanto a los sistemas de gestión de la calidad como a las BPM, al implementarlos de una manera conjunta. 
Así como la gestión del conocimiento brinda un aporte al adecuado funcionamiento de la gestión de la calidad y las BPM, también la gestión del riesgo es un facilitador para el cumplimiento de los objetivos organizacionales y de calidad cuando es enfocada en los aspectos más críticos de la organización y de los procesos de fabricación. Ella aumenta la seguridad de los pacientes y disminuye así reacciones adversas, quejas, desviaciones o retiros de producto del mercado. Un enfoque en la gestión de los riesgos permite a la empresa generar confianza dentro de la compañía y ante las entidades regulatorias, al facilitar el cumplimiento de los requisitos normativos cuando todos los procesos están alineados y las decisiones se toman después de un análisis juicioso de los riesgos que se pueden presentar desde el diseño del medicamento hasta su disposición final, aspectos también afirmados en otras investigaciones realizadas sobre la gestión del riesgo (Golaś, 2014; Charoo y Ali, 2013).

De acuerdo con todo lo anterior se recomienda lo siguiente a las organizaciones en su proceso de implementación del sistema de gestión integrado:

- Antes de decidir implementar la integración de los sistemas de gestión la alta dirección debe definir claramente el objetivo de dicha integración y el alcance que quiere dar a la gestión del riesgo y a la del conocimiento.

- Evaluar la inversión inicial de recursos económicos, tecnológicos y humanos, entre otros; realizar una proyección en el tiempo, designar específicamente los responsables de la implementación y el seguimiento y tener muy claro que el objetivo final no es la integración en sí misma, sino lograr a través de ella los objetivos organizacionales.

- En la implementación de la gestión del riesgo, tener en cuenta que el riesgo debe ser abordado no solo desde el aspecto negativo, sino también como una oportunidad que puede traer beneficios para la organización. Es necesario realizar talleres previos para desaprender este pensamiento tan arraigado en todos.

- Poner en práctica herramientas de la gestión del conocimiento y de la calidad que sean sencillas, lúdicas y que involucren a todas las personas de una manera activa. El objetivo debe ser que cada uno sienta que su aporte es valioso para la organización, y ello implica disponer de un ambiente apropiado para ir garantizando un cambio de la cultura empresarial, que requiere la implementación de la integración de los sistemas.

- La gestión del riesgo y el conocimiento para integrarse con las BPM debe cubrir especialmente el diseño y desarrollo de los medicamentos ya que es allí donde se logra obtener la mayor información y el conocimiento de aquellos. En efecto, desde esa etapa se definen las características de calidad del producto y los parámetros de operación del proceso que acompañarán toda la vida útil del medicamento pues un cambio en ese punto involucra estudios nuevos y prolongados y autorizaciones correspondientes por parte de las autoridades sanitarias.

\section{REFERENCIAS}

Babativa, O. y Hernández, M. (2011). Determinación y evaluación de la gestión del conocimiento en una organización del sector farmacéutico nacional. Signos. Investigación en Sistemas de Gestión, 1 y 2(3), 54-67.

Charoo, N. y Ali, A. (2013). Quality risk management in pharmaceutical development. Drug Development \& Industrial Pharmacy, 7 (39), 947-960. Doi: https:// doi.org/10.3109/03639045.2012.699065 
De la Hoz, J., Carrillo, E. y Gómez, L. (2012). Gestión de la calidad y del conocimiento: dos enfoques complementarios. AD-minister, 21, 71-85.

Duran, C., Çetindere, A. y Şahan, Ö. (2014). An analysis on the relationship between total quality management practices and knowledge management: The case of Eskişehir. Procedia. Social and Behavioral Sciences, 109, 65-77. Doi: https://doi.org/10.1016/j. sbspro.2013.12.422

Fábregas, A., García, E., Pérez, P., Suñé, J., Tico, J. y Miñarro, M. (2010). Quality assurance in research: Incorporating ISO 9001:2000 into a GMP quality management system in a pharmaceutical $\mathrm{R}+\mathrm{D}+\mathrm{I}$ center. Accred Qual Assur, 15, 297-304. Doi: https:// doi.org/10.1007/s00769-009-0610-z

García, A., Vallejo, D. y Mora, H. (2015). La calidad desde el diseño: principios y oportunidades para la industria farmacéutica. Estudios Gerenciales, 134(31), 68-78. Doi: https://doi.org/10.1016/j.estger.2014.09.005

Golaś, H. (2014). Risk management as part of the quality management system according to ISO 9001. Communications in Computer and Information Science, 435, 519-524. Doi: https://doi.org/10.1007/978-3319-07854-0_90

Guebitz, B., Schnedl, H. y Khinast, J. (2012). A risk management ontology for Quality-by Design based on a new development approach according GAMP 5.0. Expert Systems with Applications, 8 (39), 7291-7301. Doi: https://doi.org/10.1016/j. eswa.2012.01.089

Haleem, R., Salem, M., Fatahallah, F. y Abdelfattah, L. (2014). Quality in the pharmaceutical industry: A literature review. Saudi Pharmaceutical Journal,
23(5), 463-469. Doi: https://doi.org/10.1016/j. jsps.2013.11.004

Hernández, R., Fernández, C. y Baptista, M. (2010). Metodología de la investigación. México: McGraw-Hill Interamericana.

ICH (International Conference on Harmonisation of Technical Requirements for Registration of Pharmaceuticals for Human Use) (2005). Harmonised tripartite guideline quality risk management Q9. Ginebra: ICH.

Icontec (Instituto Colombiano de Normas Técnicas y Certificación) (2015). Sistemas de gestión de la calidad. Requisitos. NTC-ISO 9001.Bogotá: Icontec.

Icontec (Instituto Colombiano de Normas Técnicas y Certificación) (2011). Gestión del riesgo. Principios y directrices. NTC-ISO 31000. Bogotá: Icontec.

Kahreh, Z., Shirmohammadi, A. y Kahreh, M. (2014). Explanatory study towards analysis the relationship between total quality management and knowledge management. Procedia. Social and Behavioral Sciences, 109, 600-604. Doi: https://doi.org/10.1016/j. sbspro.2013.12.513

Kawai, H., Kitajima, T., Fuchino, T., Sekia, H. y Naka, Y. (2013). Quality assessment support system and its use in pharmaceutical plant operations. Computers \& Chemical Engineering, 59, 156-163. Doi: https://doi.org/10.1016/j.compchemeng.2013.06.010

Martín, M. C. (2004). Diseño y validación de cuestionario. Matronas Profesión, 17(5), 23-29.

OMS (Organización Mundial de la Salud) (1987). Anexo 1, Prácticas adecuadas de fabricación de productos 
farmacéuticas. Serie de Informes Técnicos 823. Ginebra: OMS.

OMS (Organización Mundial de la Salud) (2010). Guía de buenas prácticas de la OMS para laboratorios de control de calidad de productos farmacéuticos. Informe $n .{ }^{\circ} 44$, anexo 1 de la serie de Informes Técnicos 957. Ginebra: OMS.

Peluffo, M. y Catalán, E. (2002). Introducción a la gestión del conocimiento y su aplicación al sector público. Serie Manuales, 22. Santiago de Chile: ONU.
Poli, M., Petroni, D., Pardini, S., Salvadori, P. y Menichetti, L. (2012). Implementation of a quality assurance system according to GMP and ISO 9001: 2008 standard for radiopharmaceutical production in a public research centre. Accreditation and Quality Assurance, 3(17), 341-348. Doi: https://doi. org/10.1007/s00769-012-0877-3

Velásquez, M. (2012). Planificación de un sistema de gestión de la calidad como plataforma para integrar otros modelos de gestión (Tesis de maestría). Universidad Santo Tomás, Bogotá, Colombia.

\section{ANEXOS}

1) Operacionalización de la investigación

\begin{tabular}{|c|c|c|c|}
\hline Categoría central o familia & Categorías & Subcategorías & Código Atlas.Ti \\
\hline \multirow{13}{*}{ BPM } & \multirow{6}{*}{ Beneficios } & $\begin{array}{l}\text { Reducción de tiempos de respuesta } \\
\text { al cliente }\end{array}$ & $\begin{array}{l}\text { BPM-Beneficios-Reducción de producto no } \\
\text { conforme }\end{array}$ \\
\hline & & Optimización de recursos & BPM-Beneficios-Optimización de recursos \\
\hline & & Optimización de procesos & BPM-Beneficios-Optimización de procesos \\
\hline & & $\begin{array}{l}\text { Reducción de tiempos de respuesta } \\
\text { al cliente }\end{array}$ & $\begin{array}{l}\text { BPM-Beneficios-Reducción de tiempos de } \\
\text { respuesta al cliente. }\end{array}$ \\
\hline & & Satisfacción del cliente & BPM-Beneficios- Satisfacción del cliente \\
\hline & & Posicionamiento en el mercado & $\begin{array}{l}\text { BPM-Beneficios- Posicionamiento en el } \\
\text { mercado }\end{array}$ \\
\hline & \multirow{2}{*}{$\begin{array}{l}\text { Articulación con plataforma } \\
\text { estratégica }\end{array}$} & $\begin{array}{l}\text { Coherencia con metas, objetivos y } \\
\text { visión de la organización }\end{array}$ & $\begin{array}{l}\text { BPM-Articulación con plataforma } \\
\text { estratégica-Coherencia con metas, objetivos } \\
\text { y visión de la organización }\end{array}$ \\
\hline & & $\begin{array}{l}\text { Apoyo al cumplimiento de metas, } \\
\text { objetivos y misión de la organización }\end{array}$ & $\begin{array}{l}\text { BPM-Articulación con plataforma } \\
\text { estratégica-Apoyo al cumplimiento de } \\
\text { metas, objetivos y misión de la organización }\end{array}$ \\
\hline & \multirow{2}{*}{ Enfoque en riesgos } & $\begin{array}{l}\text { Enfoque en análisis de riesgos del } \\
\text { producto }\end{array}$ & $\begin{array}{l}\text { BPM-Enfoque en riesgos-Enfoque en } \\
\text { análisis de riesgos del producto }\end{array}$ \\
\hline & & $\begin{array}{l}\text { Enfoque en análisis de riesgos sobre } \\
\text { las personas }\end{array}$ & $\begin{array}{l}\text { BPM-Enfoque en riesgos-Enfoque en } \\
\text { análisis de riesgos sobre las personas }\end{array}$ \\
\hline & \multirow{3}{*}{ Enfoque del conocimiento } & $\begin{array}{l}\text { Cultura de buenas prácticas de } \\
\text { manufactura }\end{array}$ & $\begin{array}{l}\text { BPM-Enfoque del conocimiento-Cultura de } \\
\text { buenas prácticas de manufactura }\end{array}$ \\
\hline & & Diversificación de productos & $\begin{array}{l}\text { BPM-Enfoque del conocimiento- } \\
\text { Diversificación de productos }\end{array}$ \\
\hline & & Participación & $\begin{array}{l}\text { BPM-Enfoque del conocimiento- } \\
\text { Participación }\end{array}$ \\
\hline
\end{tabular}




\begin{tabular}{|c|c|c|c|}
\hline Categoría central o familia & Categorías & Subcategorías & Código Atlas.Ti \\
\hline \multirow{13}{*}{ Gestión de la calidad } & \multirow{2}{*}{$\begin{array}{l}\text { Metodología de la } \\
\text { implementación }\end{array}$} & $\begin{array}{l}\text { Implementación desde lo } \\
\text { documental }\end{array}$ & $\begin{array}{l}\text { SGC-Metodología de implementación- } \\
\text { Implementación desde lo documental }\end{array}$ \\
\hline & & Enfoque PHVA & $\begin{array}{l}\text { SGC-Metodología de implementación- } \\
\text { Enfoque PHVA }\end{array}$ \\
\hline & \multirow{5}{*}{ Beneficios } & $\begin{array}{l}\text { Reducción de tiempos de respuesta } \\
\text { al cliente }\end{array}$ & $\begin{array}{l}\text { SGG-Beneficios-Reducción de producto no } \\
\text { conforme }\end{array}$ \\
\hline & & Optimización de recursos & SGC-Beneficios-Optimización de recursos \\
\hline & & Optimización de procesos & SGC-Beneficios-Optimización de procesos \\
\hline & & $\begin{array}{l}\text { Reducción de tiempos de respuesta } \\
\text { al cliente }\end{array}$ & $\begin{array}{l}\text { SGG-Beneficios-Reducción de tiempos de } \\
\text { respuesta al cliente }\end{array}$ \\
\hline & & Satisfacción del cliente & SGC-Beneficios-Satisfacción del cliente \\
\hline & \multirow{3}{*}{$\begin{array}{l}\text { Articulación con plataforma } \\
\text { estratégica }\end{array}$} & $\begin{array}{l}\text { Coherencia con metas, objetivos y } \\
\text { visión de la organización }\end{array}$ & $\begin{array}{l}\text { SGG-Articulación con plataforma } \\
\text { estratégica-Coherencia con metas, objetivos } \\
\text { y visión de la organización }\end{array}$ \\
\hline & & $\begin{array}{l}\text { Apoyo al cumplimiento de metas, } \\
\text { objetivos y misión de la organización }\end{array}$ & $\begin{array}{l}\text { SGG-Articulación con plataforma } \\
\text { estratégica-Apoyo al cumplimiento de } \\
\text { metas, objetivos y misión de la organización }\end{array}$ \\
\hline & & $\begin{array}{l}\text { Seguimiento al cumplimiento de } \\
\text { metas, objetivos y misión de la } \\
\text { organización }\end{array}$ & $\begin{array}{l}\text { SGG-Articulación con plataforma } \\
\text { estratégica-Seguimiento al cumplimiento de } \\
\text { metas, objetivos y misión de la organización }\end{array}$ \\
\hline & Integración con BPM & Ventajas & SGG-Integración con BPM-Ventajas \\
\hline & \multirow{2}{*}{ Enfoque del conocimiento } & Aprendizaje organizacional & $\begin{array}{l}\text { SGG-Gestión del conocimiento-Aprendizaje } \\
\text { organizacional }\end{array}$ \\
\hline & & Difusión & SGC-Gestión del conocimiento-Difusión \\
\hline \multirow{7}{*}{ Riesgo } & \multirow{3}{*}{$\begin{array}{l}\text { En los modelos del SGC } \\
\text { y BPM }\end{array}$} & Enfoque preventivo & $\begin{array}{l}\text { RIESGOS-En los modelos del SGC y } \\
\text { BPM-Enfoque preventivo }\end{array}$ \\
\hline & & Enfoque correctivo & $\begin{array}{l}\text { RIESGOS-En los modelos del SGC y } \\
\text { BPM-Enfoque correctivo }\end{array}$ \\
\hline & & $\begin{array}{l}\text { Enfoque sobre la toma de decisiones } \\
\text { en los resultados }\end{array}$ & $\begin{array}{l}\text { RIESGOS-En los modelos del SGC y } \\
\text { BPM-Enfoque sobre la toma de decisiones } \\
\text { en los resultados }\end{array}$ \\
\hline & \multirow{2}{*}{$\begin{array}{l}\text { Articulación con plataforma } \\
\text { estratégica }\end{array}$} & $\begin{array}{l}\text { Transversalidad en todos los } \\
\text { procesos }\end{array}$ & $\begin{array}{l}\text { RIESGOS-Articulación con plataforma } \\
\text { estratégica-Transversalidad en todos los } \\
\text { procesos }\end{array}$ \\
\hline & & $\begin{array}{l}\text { Estrategias para evitar, mitigar } 0 \\
\text { potencializar }\end{array}$ & $\begin{array}{l}\text { RIESGOS-Articulación con plataforma } \\
\text { estratégica-Estrategias para evitar, mitigar o } \\
\text { potencializar }\end{array}$ \\
\hline & \multirow{2}{*}{ Comunicación } & Difusión del análisis & $\begin{array}{l}\text { RIESGOS-Comunicación-Difusión del } \\
\text { análisis }\end{array}$ \\
\hline & & Difusión de resultados & $\begin{array}{l}\text { RIESGOS-Comunicación-Difusión de } \\
\text { resultados }\end{array}$ \\
\hline
\end{tabular}


Integración de la gestión de la calidad con las buenas prácticas de manufactura en tres empresas farmacéuticas de inyectables en Bogotá, D. C

\begin{tabular}{|c|c|c|c|}
\hline Categoría central o familia & Categorías & Subcategorías & Código Atlas.Ti \\
\hline \multirow{10}{*}{ Gestión del conocimiento } & \multirow{2}{*}{$\begin{array}{l}\text { Procesamiento del } \\
\text { conocimiento }\end{array}$} & Herramientas & $\begin{array}{l}\text { GC-Procesamiento del conocimiento- } \\
\text { Herramientas }\end{array}$ \\
\hline & & Debilidades & $\begin{array}{l}\text { GC-Procesamiento del conocimiento- } \\
\text { Debilidades }\end{array}$ \\
\hline & \multirow{3}{*}{$\begin{array}{l}\text { Gestión del talento } \\
\text { humano }\end{array}$} & Aporte & GC-Gestión del talento humano-Aporte \\
\hline & & Selección y promoción & $\begin{array}{l}\text { GC-Gestión del talento humano-Selección } \\
\text { y promoción }\end{array}$ \\
\hline & & Competencias & $\begin{array}{l}\text { GC-Gestión del talento humano- } \\
\text { Competencias }\end{array}$ \\
\hline & \multirow{2}{*}{ Difusión } & Formación & GC-Difusión-Formación \\
\hline & & Recursos & GC-Difusión-Recursos \\
\hline & \multirow{3}{*}{ Beneficios } & $\begin{array}{l}\text { Oportunidades para el desempeño } \\
\text { organizacional }\end{array}$ & $\begin{array}{l}\text { GG-Beneficios-Oportunidades para el } \\
\text { desempeño organizacional }\end{array}$ \\
\hline & & Generación de valor & GG-Beneficios-Generación de valor \\
\hline & & Éxito organizacional & GC-Beneficios-Éxito organizacional \\
\hline
\end{tabular}

2) Tabla de correspondencias de requisitos de cada sistema o herramienta de gestión basada en el ciclo PHVA.

\begin{tabular}{|l|}
\hline Planear \\
\hline Hacer \\
\hline Verificar \\
\hline Actuar \\
\hline
\end{tabular}

Tabla de correspondencias de requisitos de cada sistema o herramienta de gestión basado en el ciclo PHVA

\begin{tabular}{|l|l|l|l|}
\hline \multicolumn{1}{|c|}{ ISO 9001: 2015 } & \multicolumn{1}{|c|}{ BPM (Informe 32 de la OMS) } & \multicolumn{1}{c|}{ ISO 31000:2011 } & $\begin{array}{l}\text { Gestión del conocimiento (Documen- } \\
\text { to de la ONU, Peluffo y Catalán) }\end{array}$ \\
\hline $\begin{array}{l}\text { 4. CONTEXTO DE LA } \\
\text { ORGANIZACIÓN }\end{array}$ & NO SE EVIDENCIA & $\begin{array}{l}\text { 4. MARCO DE } \\
\text { REFERENCIA }\end{array}$ & 2. DIAGNÓSTICO INICIAL \\
\hline $\begin{array}{l}\text { 4.1 Comprensión de la } \\
\text { organización y su contexto }\end{array}$ & NO SE EVIDENCIA & 4.1 Generalidades & 2.1 Mapa de conocimiento organizacional \\
\hline $\begin{array}{l}\text { 4.2 Comprensión de las } \\
\text { necesidades y expectativas de } \\
\text { las partes interesadas }\end{array}$ & NO SE EVIDENCIA & $\begin{array}{l}5.3 \text { Establecimiento del } \\
\text { contexto }\end{array}$ & 2.2 Diagnóstico de prácticas habituales \\
\hline $\begin{array}{l}\text { 4.3 Determinación del alcance } \\
\text { del SGC }\end{array}$ & $\begin{array}{l}\text { PRIMERA PARTE: } \\
\text { Administración de la calidad en la } \\
\text { industria farmacéutica, filosofía y } \\
\text { elementos esenciales }\end{array}$ & $\begin{array}{l}4.3 \text { Diseño del marco de } \\
\text { referencia para la gestión del } \\
\text { riesgo }\end{array}$ & NO SE EVIDENCIA \\
\hline 4.4 SGC y sus procesos & $\begin{array}{l}\text { 1. GARANTÍA DE LA } \\
\text { CALIDAD. } \\
\text { 2. BUENAS PRÁCTICAS } \\
\text { DE MANUFACTURA DE } \\
\text { PRODUCTOS }\end{array}$ & $\begin{array}{l}\text { 4.4 Implementar la gestión } \\
\text { del riesgo }\end{array}$ & NO SE EVIDENCIA \\
\hline
\end{tabular}




\begin{tabular}{|c|c|c|c|}
\hline ISO 9001: 2015 & BPM (Informe 32 de la OMS) & ISO 31000:2011 & $\begin{array}{l}\text { Gestión del conocimiento (Documen- } \\
\text { to de la ONU, Peluffo y Catalán) }\end{array}$ \\
\hline 5. LIDERAZGO & NO SE EVIDENCIA & NO SE EVIDENCIA & NO SE EVIDENCIA \\
\hline 5.1 Liderazgo y compromiso & NO SE EVIDENCIA & 4.2 Dirección y compromiso & NO SE EVIDENCIA \\
\hline 5.2 Política & NO SE EVIDENCIA & NO SE EVIDENCIA & NO SE EVIDENCIA \\
\hline \begin{tabular}{|l|}
5.3 Roles, responsabilidades y \\
autoridades de la organización
\end{tabular} & 10. PERSONAL & NO SE EVIDENCIA & NO SE EVIDENCIA \\
\hline \multicolumn{4}{|l|}{ 6. PLANIFICACIÓN } \\
\hline $\begin{array}{l}\text { 6.1 Acciones para abordar } \\
\text { riesgos y oportunidades }\end{array}$ & NO SE EVIDENCIA & 5. PROCESO & $\begin{array}{l}\text { 2.3 Evaluación de las capacidades } \\
\text { dinámicas de la organización }\end{array}$ \\
\hline $\begin{array}{l}\text { 6.2 Objetivos de la calidad y } \\
\text { planificación para lograrlos }\end{array}$ & NO SE EVIDENCIA & 5.1 Generalidades & $\begin{array}{l}\text { 3. DEFINICIÓN DE LOS } \\
\text { OBJETIVOS DEL CONOCIMIENTO }\end{array}$ \\
\hline $\begin{array}{l}\text { 6.3 Planificación de los } \\
\text { cambios }\end{array}$ & NO SE EVIDENCIA & NO SE EVIDENCIA & NO SE EVIDENCIA \\
\hline 7. APOYO & NO SE EVIDENCIA & NO SE EVIDENCIA & NO SE EVIDENCIA \\
\hline 7.1 Recursos & NO SE EVIDENCIA & NO SE EVIDENCIA & NO SE EVIDENCIA \\
\hline 7.2 Competencia & NO SE EVIDENCIA & NO SE EVIDENCIA & $\begin{array}{l}\text { 2.3 Evaluación de las capacidades } \\
\text { dinámicas de la organización }\end{array}$ \\
\hline 7.3 Toma de conciencia & NO SE EVIDENCIA & NO SE EVIDENCIA & NO SE EVIDENCIA \\
\hline 7.4 Comunicación & NO SE EVIDENCIA & 5.2 Comunicación y consulta & NO SE EVIDENCIA \\
\hline 7.5 Información documentada & 14. DOCUMENTACIÓN & $\begin{array}{l}5.7 \text { Registro proceso para la } \\
\text { gestión riesgo }\end{array}$ & 5.1 Codificación de documentos \\
\hline 8. OPERACIÓN & $\begin{array}{l}\text { 15. BUENAS PRÁCTICAS DE } \\
\text { PRODUCGIÓN Y CONTROL } \\
\text { DE CALIDAD }\end{array}$ & \multirow{8}{*}{$\begin{array}{l}5.4 \text { Valoración del riesgo } 5.5 \\
\text { Tratamiento del riesgo }\end{array}$} & \multirow{8}{*}{$\begin{array}{l}\text { 4. PRODUCGIÓN DE } \\
\text { CONOCIMIENTO } \\
\text { ORGANIZACIONAL. } \\
\text { 5. ALMACENAJE Y } \\
\text { ACTUALIZACIÓN. } \\
\text { 6. CIRCULACIÓN Y UTILIZACIÓN } \\
\text { DE LOS CONOCIMIENTOS: LOS } \\
\text { USUARIOS }\end{array}$} \\
\hline $\begin{array}{l}\text { 8.1 Planificación y control } \\
\text { operacional }\end{array}$ & 5. VALIDACIÓN & & \\
\hline $\begin{array}{l}\text { 8.2 Requisitos para los } \\
\text { productos y servicios }\end{array}$ & 3. CONTROL DE CALIDAD & & \\
\hline $\begin{array}{l}8.3 \text { Diseño y desarrollo de los } \\
\text { productos y servicios }\end{array}$ & $\begin{array}{l}\text { 4. SANEAMIENTO E HIGIENE } \\
\text { 11. INSTALACIONES. } \\
\text { 12. EQUIPOS. } \\
\text { 13. MATERIALES } \\
\end{array}$ & & \\
\hline $\begin{array}{l}\text { 8.4 Control de los procesos, } \\
\text { productos y servicios } \\
\text { suministrados externamente }\end{array}$ & $\begin{array}{l}\text { 8. PRODUCCIÓN Y ANÁLISIS } \\
\text { POR CONTRATO }\end{array}$ & & \\
\hline $\begin{array}{l}\text { 8.5 Producción y provisión del } \\
\text { servicio }\end{array}$ & \begin{tabular}{|l} 
17. PRODUCTOS \\
FARMACÉUTICOS \\
ESTÉRILES \\
\end{tabular} & & \\
\hline \begin{tabular}{|l|}
8.6 Liberación de los productos \\
y servicios
\end{tabular} & 3. CONTROL DE CALIDAD & & \\
\hline $\begin{array}{l}8.7 \text { Control de las salidas no } \\
\text { conformes }\end{array}$ & $\begin{array}{l}\text { 6. QUEJAS } \\
\text { 7. RETIRO DE PRODUCTO } \\
\text { DEL MERCADO }\end{array}$ & & \\
\hline
\end{tabular}


Integración de la gestión de la calidad con las buenas prácticas de manufactura en tres empresas farmacéuticas de inyectables en Bogotá, D. C

\begin{tabular}{|c|c|c|c|}
\hline ISO 9001: 2015 & BPM (Informe 32 de la OMS) & ISO 31000:2011 & $\begin{array}{c}\text { Gestión del conocimiento (Documen- } \\
\text { to de la ONU, Peluffo y Catalán) }\end{array}$ \\
\hline \multicolumn{4}{|c|}{ 9. EVALUACIÓN DEL DESEMPEÑO } \\
\hline $\begin{array}{l}\text { 9.1 Seguimiento, medición, } \\
\text { análisis y evaluación }\end{array}$ & NO SE EVIDENCIA & $\begin{array}{l}\text { 4.5 Monitorear y revisar el } \\
\text { marco de referencia }\end{array}$ & \multirow{2}{*}{ 7. MEDICIÓN DEL DESEMPEÑO } \\
\hline 9.2 Auditoría interna & $\begin{array}{l}\text { 9. AUTOINPECCIÓN Y } \\
\text { AUDITORÍAS DE CALIDAD }\end{array}$ & 5.6 Monitoreo y revisión & \\
\hline 9.3 Revisión por la dirección & NO SE EVIDENCIA & NO SE EVIDENCIA & NO SE EVIDENCIA \\
\hline \multicolumn{4}{|l|}{ 10. MEJORA } \\
\hline 10.1 Generalidades & NO SE EVIDENCIA & $\begin{array}{l}\text { 4.6 Mejora continua del marco } \\
\text { de referencia }\end{array}$ & NO SE EVIDENCIA \\
\hline $\begin{array}{l}10.2 \text { No conformidad y acción } \\
\text { correctiva }\end{array}$ & NO SE EVIDENCIA & NO SE EVIDENGIA & NO SE EVIDENCIA \\
\hline 10.3 Mejora Continua & NO SE EVIDENCIA & NO SE EVIDENCIA & NO SE EVIDENCIA \\
\hline
\end{tabular}

